\title{
Dynamic Trajectory Generation for Serial Elastic Actuated Robots *
}

\author{
Florian Petit* Dominic Lakatos* Werner Friedl * \\ Alin Albu-Schäffer*
}

\author{
* Institute of Robotics and Mechatronics, German Aerospace Center \\ (DLR), D-82234 Oberpfaffenhofen, Germany (e-mail: \{florian.petit, \\ dominic.lakatos, werner.friedl, alin.albu-schaeffer\}@dlr.de).
}

\begin{abstract}
Robotic systems can benefit from the introduction of properly chosen joint elasticity. Besides their robustness against rigid impact, the energy saving capabilities may increase the system dynamics. In this paper, a method applicable for robots with serial elastic joints is presented, which embodies a desired oscillatory behavior into the hardware and thereby leads to improved performance. This is achieved by shaping the flexible joint robot as a linear onemode system and embodying the natural frequency of the real intrinsic behavior. An algorithm is presented for shaping the one-mode property and exciting the system via a negative definite damping term in a decoupled coordinate space. The output of the approach is a dynamic trajectory resulting in a coordinated link motion and synchronized transfer of kinetic and potential energy. Furthermore, the dynamic trajectory is commanded to the real robot via a motor PD controller, where asymptotic stability for both subsystems - i.e. the trajectory generator and the controlled robot-is proven.

The method is validated on a two-link serial elastic actuated robot. Both, simulation and experiment confirm the eigenmode embodiment, energy efficiency by velocity enlargement between motor and link side motion, and synchronized joint motion.
\end{abstract}

Keywords: Serial elastic actuated, nonlinear oscillations, modal decoupling, singular perturbation.

\section{INTRODUCTION}

Actuators with intrinsic compliances promise several benefits for a variety of robotic systems. Besides of technological considerations like the use of the known force-deflection relation to estimate joint torques (Pratt and Williamson (1995)), especially the increased mechanical robustness is a major advantage: The spring elements act as low pass filters against peak torques as they may occur during rigid impacts. This is relevant for scenarios like a robotic hand manipulating objects (Grebenstein et al. (2011) describe such a system) or a running robot in ground contact (Raibert (1986) makes use of passive elasticities in running robots). Furthermore, the energy storage properties of passive compliance are interesting and could be beneficial for solving highly dynamical tasks such as fast point-topoint movements, bipedal walking, throwing etc. Some work has been done to exploit the elastic elements to gain increased end effector velocity (Braun et al. (2011); Haddadin et al. (2012)) or tune the resulting oscillatory behaviour to a predefined trajectory (Uemura and Kawamura (2009); Visser et al. (2011)). The mentioned work mainly uses optimization and iterative methods to adjust the joint stiffness and torque to achieve the desired action. Furthermore, the topic of trajectory tracking has been addressed in multiple ways. Ranging from input shaping

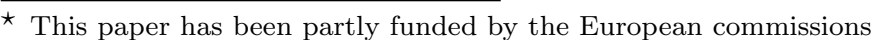
Seventh Framework Program as part of the project VIACTORS under grant No. 231554.
}

techniques, optimal control, and optimization based trajectory generation to adaptive control and flatness based feed forward command generation, many approaches have been developed. However, the presented approach focuses on exploiting the natural system dynamics.

This work aims to exploit the joint compliance of multiple degrees of freedom (DoF) series elastic actuated (SEA) robots by identifying and shaping intrinsic resonance properties of the system. The goal is to synchronize the motions of the complete system by coordinating the motion of the single joints. Since synchronized motion induces synchronized transfer between potential and kinetic energy, there exist a point where the Hamiltonian energy is completely kinetic. Such a property can then be exploited while solving a highly dynamical periodic task.

In order to reach the desired behavior an eigenmode analysis is performed on the locally linearized robot model. Then a desired system is formulated such that all eigenmodes of the resulting dynamics are the same. To achieve the desired dynamics, the robot needs to be tuned e.g. by varying the joint stiffnesses, or by an active control algorithm as it is given in the following.

Furthermore, an efficient way to exploit the one-mode dynamics is presented. Motivated by linear second order systems, a negative damping coefficient is used to excite the system and obtain controlled oscillatory behaviour. The excitation acting at the natural frequency of the 
system ensures the highest possible input/output gain and thereby high energy input/output.

Finally, the algorithm is adapted for the use with series elastic actuated robots which can be modeled by a reduced flexible joint model. The approach allows to deal with non-linearities as they are generated by the rigid robot dynamics. Simulations and experiments on a planar double pendulum robot show the effectiveness of the approach.

An advantage of the procedure is the possibility to analyze the embodiment of the resonance motion by simply comparing the desired and the passive system dynamics. An important finding of the analysis is that coupling stiffnesses are necessary to achieve the one-mode property as a result of the coupled mass matrix. This is also observed by Haddadin et al. (2012). Additionally, these coupling stiffnesses are the counterpart of bi-articulated muscles in the human body.

The paper is organized as follows. First, a short overview of the types of flexible systems which can be used with the approach is presented in Section 2. Section 3 presents the approach for the eigenmode analysis and shaping of the simplified linearized dynamics and the procedure for exciting the desired system. Section 4 contains three parts. First, the approach is extended for the use with a flexible joint robot with nonlinear dynamics (i.e. nonlinear mass matrix and Coriolis/centrifugal forces). Then the stability of the algorithm is analyzed. Finally, two trajectory embodiment measures are introduced for evaluating the choice of the intrinsic mechanical stiffness. In Section 5 simulations and experimental results are presented. Here, the range and performance of the approach is analyzed. Section 6 summarizes the work, and gives an outlook.

\section{SYSTEM DESCRIPTION}

Before the details of the approach are presented, a short overview of the passive elastic systems which can be tackled is given.

The use of flexibilities in robotic actuators has been explored e.g. by Pratt and Williamson (1995) in their work on Series Elastic Actuators. Although not limited to, the series elasticity used in their setup shows a constant spring rate and one joint can be modeled by

$$
\begin{array}{r}
M \ddot{q}=K(\theta-q) \\
B \ddot{\theta}+K(\theta-q)=\tau_{m},
\end{array}
$$

where $\theta$ and $q$ represent the motor and link position, $B$ and $M$ are the motor and link inertia, $K$ is a constant spring rate, and $\tau_{m}$ is the motor torque. This model is similar to the reduced flexible joint model described in Spong (1987). Due to the constant spring rate, these SEAs show a force bandwidth limitation.

Recent developments aim to overcome the force bandwidth limitation by introducing a variable stiffness spring in series (Tonietti et al. (2005); Wolf and Hirzinger (2008)). Several actuators can be modeled by a similar formulation as (1) (Jafari et al. (2011)):

$$
\begin{array}{r}
M \ddot{q}=K(\sigma)(\theta-q) \\
B \ddot{\theta}+K(\sigma)(\theta-q)=\tau_{m},
\end{array}
$$

where $\sigma$ is a stiffness variation parameter, realized by an additional actuator. This model describes an actuator with linear, variable series elasticity $K(\sigma)$, where for a fixed stiffness variation parameter $\sigma$ the model (1) is equivalent (cf. Spong (1987)). A generalized model for variable stiffness actuators was proposed in Albu-Schäffer et al. (2010). The main difference is, that the joint torque itself may be of non-linear character. The model is given by

$$
\begin{array}{r}
M \ddot{q}=\Psi(\theta-q, \sigma) \\
B \ddot{\theta}+\Psi(\theta-q, \sigma)=\tau_{m},
\end{array}
$$

where $\Psi(\theta-q, \sigma)$ is a general non-linear function depending on the link deflection $\theta-q$ and the stiffness variation parameter $\sigma$.

The following theory is applicable for joints which can be modeled by (1). The non-linear behaviour of such multi joint robots (Brooks et al. (1999)) stems not from the joint flexibility, but form other non-linearities like the mass dynamics. However the presented theory can be used to adjust the stiffness parameter of joints of type (2) to adapt the passive dynamics of the system. The high stiffness changes of joints of type (3) with the stiffness changing up to several $1000 \%$ permits to use the presented approach.

\section{EIGENMODE SHAPING AND SYSTEM EXCITATION}

The presented method is based on linear oscillation theory, and will be introduced for an idealized system. Therefore, the homogeneous second order linear differential equation

$$
\boldsymbol{M} \ddot{\boldsymbol{q}}+\boldsymbol{K}_{d} \boldsymbol{q}=\mathbf{0}
$$

is considered as the linearized model of the multi-joint robot manipulator, where the linearization is around an equilibrium position. In (4) $\boldsymbol{q} \in \mathbb{R}^{n}$ is the vector of the $n$ joint coordinates, and $\boldsymbol{M} \in \mathbb{R}^{n \times n}$ and $\boldsymbol{K}_{d} \in \mathbb{R}^{n \times n}$ are the constant, positive definite, and symmetric mass matrix and stiffness matrix, respectively.

\subsection{Eigenmode shaping}

In a first step, the eigenmodes of the system (4) are chosen such that $n$ repeated eigenvalues arise. Therefore the general eigenvalue problem

$$
\boldsymbol{K}_{d} \hat{\boldsymbol{q}}=\lambda \boldsymbol{M} \hat{\boldsymbol{q}}
$$

is considered. From (5) the following condition is deduced: for $i=1,2, \ldots, n$ there exist a pair $\left(\lambda_{i}, \hat{\boldsymbol{q}}_{i}\right)$, such that

$$
\boldsymbol{K}_{d} \hat{\boldsymbol{q}}_{i}=\lambda_{i} \boldsymbol{M} \hat{\boldsymbol{q}}_{i} \text {. }
$$

Since $n$ identical eigenvalues are claimed, we can rewrite

$$
\boldsymbol{K}_{d} \boldsymbol{Q}=\lambda_{0} \boldsymbol{M} \boldsymbol{Q}
$$

where $\boldsymbol{Q}=\left(\hat{\boldsymbol{q}}_{1}, \hat{\boldsymbol{q}}_{2}, \ldots, \hat{\boldsymbol{q}}_{n}\right)$; consequently the system (4) is shaped in one eigenmode, if the stiffness matrix is proportional to the mass matrix, i.e.

$$
\boldsymbol{K}_{d}=\lambda_{0} \boldsymbol{M} \text {. }
$$

This implies that $\boldsymbol{Q}$ is regular.

\subsection{System excitation}

The system (4) where condition (8) holds consists of only first modes. To obtain an oscillatory motion, energy needs 
to be injected into the system. Therefore, a damping term will be introduced, which leads to

$$
\boldsymbol{M} \ddot{\boldsymbol{q}}+\boldsymbol{D}_{d} \dot{\boldsymbol{q}}+\boldsymbol{K}_{d} \boldsymbol{q}=\mathbf{0} .
$$

By setting the damping matrix $\boldsymbol{D}_{d} \in \mathbb{R}^{n \times n}$ to be negative or positive definite, the system energy will be increased or decreased, respectively.

To excite the DoF's of the system synchronously in resonance, the damping matrix is designed in decoupled modal coordinates. Therefore, the similarity transformation is applied to the mass matrix, i.e.

$$
\boldsymbol{Q}^{T} \boldsymbol{M} \boldsymbol{Q}=\boldsymbol{I}
$$

where $\boldsymbol{Q}$ is non-singular ${ }^{1}$. For the case of the one eigenmode system it results with (8), that

$$
\boldsymbol{Q}^{T} \boldsymbol{K}_{d} \boldsymbol{Q}=\lambda_{0} \boldsymbol{Q}^{T} \boldsymbol{M} \boldsymbol{Q}=\lambda_{0} \boldsymbol{I},
$$

and thus the modal matrix $\boldsymbol{Q}$ decouples the system (9). Finally, the damping matrix in coupled joint coordinates is obtained by

$$
\boldsymbol{D}_{d}=2 \boldsymbol{Q}^{-T} \operatorname{diag}\left(\xi_{i} \sqrt{\lambda_{0}}\right) \boldsymbol{Q}^{-1} .
$$

By choosing the damping factors $\xi_{i}=\xi_{1}=\ldots=\xi_{n}=\xi$ to be negative, the damping matrix $\boldsymbol{D}_{d}$ becomes negative definite and all DoF's of the system (9) will be excited simultaneously.

\section{ONE-MODE SHAPING FOR THE NONLINEAR REDUCED FLEXIBLE JOINT MODEL}

In the following, the linear eigenmode approach presented in Section 3 will be applied to the case of a serial elastic actuated robot manipulator. Therefore it is assumed that the manipulator dynamics can be modeled by the reduced flexible joint model (cf. (1)) proposed by Spong (1987):

$$
\begin{aligned}
\boldsymbol{M}(\boldsymbol{q}) \ddot{\boldsymbol{q}}+\boldsymbol{c}(\boldsymbol{q}, \dot{\boldsymbol{q}}) & =\boldsymbol{\tau} \\
\boldsymbol{B} \ddot{\boldsymbol{\theta}}+\boldsymbol{\tau} & =\boldsymbol{\tau}_{m} \\
\boldsymbol{\tau} & =\boldsymbol{K}(\boldsymbol{\theta}-\boldsymbol{q})+\boldsymbol{D}(\dot{\boldsymbol{\theta}}-\dot{\boldsymbol{q}})
\end{aligned}
$$

Here, $\boldsymbol{q} \in \mathbb{R}^{n}$ and $\boldsymbol{\theta} \in \mathbb{R}^{n}$ are the vectors of link and motor positions, respectively. $\boldsymbol{M}(\boldsymbol{q}) \in \mathbb{R}^{n \times n}$ is the link side mass matrix, and $\boldsymbol{c}(\boldsymbol{q}, \dot{\boldsymbol{q}}) \in \mathbb{R}^{n}$ is the link side Coriolis/centrifugal vector. $\boldsymbol{B} \in \mathbb{R}^{n \times n}$ is the constant and diagonal matrix of motor inertias, and $\boldsymbol{K}$ and $\boldsymbol{D}$ are the constant and diagonal matrices describing the joint visco elasticities.

The structure of the system (13)-(15) differs substantially from the linear one-mode dynamics (9). Thus, a dynamic trajectory generation algorithm will be presented, which shapes the desired form. Therefore the complete system will be introduced as two serially interconnected subsystems (see, Figure 1). Then, asymptotic stability of each subsystem will be proven. Finally we propose a law to embody intrinsic system properties and evaluate the resulting performance.

\subsection{Trajectory generation}

The trajectory shaping of the system (13)-(15) to a onemode dynamics is generated in two steps. In a first step

\footnotetext{
1 If $M$ is symmetric and positive definite, there exists always a nonsingular matrix $\boldsymbol{Q}$, such that $\boldsymbol{Q}^{T} \boldsymbol{M} \boldsymbol{Q}=\boldsymbol{I}$. Notice, $\boldsymbol{Q}$ is not unique. Here we normalize w.r.t. $M$.
}

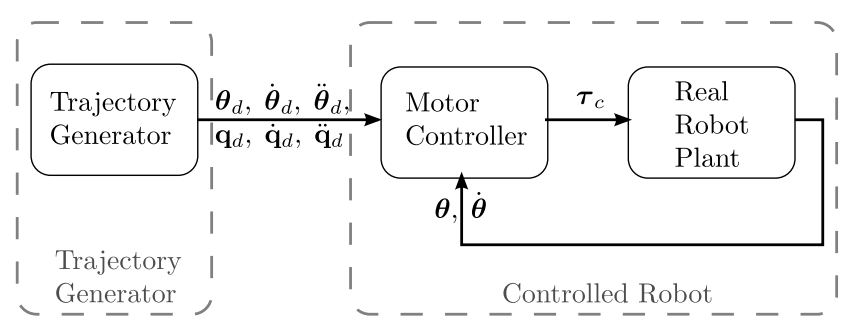

Fig. 1. Structure of the complete system: trajectory generator, motor PD controller and real robot plant. Two uncoupled subsystems are marked.

the linearization of (13) in combination with (9) gives the desired dynamics

$$
\boldsymbol{M}\left(\boldsymbol{q}_{0}\right) \ddot{\boldsymbol{q}}+\boldsymbol{D}_{d}\left(\boldsymbol{q}_{0}\right) \dot{\boldsymbol{q}}+\boldsymbol{K}_{d}\left(\boldsymbol{q}_{0}\right) \boldsymbol{q}=0 .
$$

Herein, $\boldsymbol{q}_{0} \equiv \boldsymbol{q}\left(t_{0}\right)$ denotes the instantaneous linearization about the actual link position. The solution results in the desired trajectory $\boldsymbol{q}_{d}(t)$. In the second step, $\boldsymbol{q}_{d}(t)$ is filtered by

$$
\boldsymbol{D} \dot{\boldsymbol{\theta}}_{d}+\boldsymbol{K} \boldsymbol{\theta}_{d}=\left(\boldsymbol{D}-\boldsymbol{D}_{d}\right) \dot{\boldsymbol{q}}_{d}+\left(\boldsymbol{K}-\boldsymbol{K}_{d}\right) \boldsymbol{q}_{d}+\boldsymbol{c}\left(\boldsymbol{q}_{d}, \dot{\boldsymbol{q}}_{d}\right)
$$

to obtain the motor position trajectory $\boldsymbol{\theta}_{d}(t)$.

By solving the filter dynamics (17) for the desired joint torques,

$$
\begin{aligned}
\boldsymbol{\tau}=\boldsymbol{\tau}_{d} & =\boldsymbol{K}\left(\boldsymbol{\theta}_{d}-\boldsymbol{q}_{d}\right)+\boldsymbol{D}\left(\dot{\boldsymbol{\theta}_{d}}-\dot{\boldsymbol{q}_{d}}\right) \\
& =-\boldsymbol{D}_{d}\left(\boldsymbol{q}_{0}\right) \dot{\boldsymbol{q}}_{d}-\boldsymbol{K}_{d}\left(\boldsymbol{q}_{0}\right) \boldsymbol{q}_{d}+\boldsymbol{c}\left(\boldsymbol{q}_{d}, \dot{\boldsymbol{q}}_{d}\right),
\end{aligned}
$$

it can be seen that the trajectory $\boldsymbol{\theta}_{d}(t)$ cancels out the Coriolis/centrifugal forces in the link side dynamics (13). Assuming that the motor side dynamics are sufficiently fast $^{2}$, the desired form of (16) can be obtained by following the desired trajectory $\boldsymbol{\theta}_{d}(t)$.

Compared to a design procedure as backstepping (cf. Nicosia and Tomei (1992); Oh and Lee (1997, 1998)) the measured link positions are not used to compute the motor trajectories. To prove stability of the complete system, this will be of major importance.

\subsection{Stability analysis of the complete system}

The dynamic trajectory shaping for the one-mode behavior is in coordinates of the motor position, which is not a control input of the real robot plant (13)-(15). In order to track the desired trajectory precisely, a motor $\mathrm{PD}$ controller of the form

$$
\begin{aligned}
& \boldsymbol{\tau}_{c}=\boldsymbol{B} \ddot{\boldsymbol{\theta}}_{d}-\boldsymbol{K}_{P} \tilde{\boldsymbol{\theta}}-\boldsymbol{K}_{D} \dot{\tilde{\boldsymbol{\theta}}}+\boldsymbol{\tau}_{v} \\
& \boldsymbol{\tau}_{v}=\boldsymbol{M}\left(\boldsymbol{q}_{d}\right) \ddot{\boldsymbol{q}}_{d}+\boldsymbol{c}\left(\boldsymbol{q}_{d}, \dot{\boldsymbol{q}}_{d}\right)
\end{aligned}
$$

is considered. Here $\tilde{\boldsymbol{\theta}}=\boldsymbol{\theta}-\boldsymbol{\theta}_{d}$ is the control error, $\boldsymbol{\tau}_{v}$ a torque feed forward term, and $\boldsymbol{K}_{P} \in \mathbb{R}^{n \times n}$ and $\boldsymbol{K}_{D} \in \mathbb{R}^{n \times n}$ are the constant, symmetric and positive definite controller gain matrices, respectively.

The complete approach can be divided in three units: a trajectory generator, a motor PD controller and the real robot plant. Figure 1 depicts the structure. By treating the PD controller and plant as one unit, two decoupled subsystems can be identified. This partitioning permits to analyze the stability of each subsystem separately, where

\footnotetext{
2 More precisely we suppose that $\boldsymbol{\theta}_{d} \equiv \boldsymbol{\theta}$ for all time, i.e. the singular perturbation assumption (see e.g. Kokotovic et al. (1986); Khalil (2002)) holds.
} 
stability of the complete system can be deduced, if both serially interconnected subsystems for itself are stable.

As generally known (see, e.g. Albu-Schäffer and Hirzinger (2000); De Luca et al. (2005)), the motor PD controller stabilizes the flexible joint system (13)-(15) by local asymptotic convergence. In the following the stability of the trajectory generator is analyzed.

The closed loop dynamics (16) can be transformed into modal coordinates:

$$
\ddot{z}+2 \operatorname{diag}\left(\xi_{i} \sqrt{\lambda_{0}}\right) \dot{z}+\lambda_{0} \boldsymbol{z}=\mathbf{0},
$$

where $\boldsymbol{z}=\boldsymbol{Q}^{-1} \boldsymbol{q}$. This leads to $n$ linear second order differential equations, where global asymptotic stability for the equilibrium point $(\boldsymbol{z}, \dot{\boldsymbol{z}})=\mathbf{0}$ (and consequently $(\boldsymbol{q}, \dot{\boldsymbol{q}})=\mathbf{0})$ is given. Notice, stability for the trajectory generator implies $\xi_{i}>0$. As long as oscillations are excited, i.e., $\xi_{i}<0$ it is not possible to proof stability; in that case the analytical solution of (21) can be taken into account to bound the oscillation amplitudes.

\subsection{Performance of the shaped one-mode dynamics}

The approach is useful only when the one-mode dynamics is "close" to the natural system (13)-(15), and thereby embodied into the system. This can be achieved by choosing the system parameter $\lambda_{0}$ to be the first eigenvalue of the intrinsic system, i.e.

$$
\lambda_{0}=\min \left(\operatorname{eig}\left(\boldsymbol{K}, \boldsymbol{M}\left(\boldsymbol{q}_{0}\right)\right)\right)=\text { const. }
$$

In order to quantify the difference between the natural and the shaped system we propose two evaluation criteria: the stiffness embodiment number and the motion embodiment number. Therefore an oscillation period $T=t_{2}-t_{1}$, where $t_{1}<t_{2}$ is an arbitrary instant of time, is observed.

Since the passive joint stiffness $\boldsymbol{K}$ differs from the shaped stiffness matrix $\boldsymbol{K}_{d}$, the effective stiffness has to be altered. To measure the closeness of the passive elasticity to the desired stiffness, we propose the stiffness embodiment number

$$
\beta=\left(\int_{t_{1}}^{t_{2}}\left\|\boldsymbol{K}-\boldsymbol{K}_{d}(t)\right\|_{F} \mathrm{~d} t\right) /\left(\int_{t_{1}}^{t_{2}}\|\boldsymbol{K}\|_{F} \mathrm{~d} t\right) .
$$

This number can be seen as a measure of the loss in performance due to stiffness shaping. Here $\|\cdot\|_{F}$ computes the Frobenius norm of a matrix.

The motion embodiment number is proposed as

$$
\alpha=\left(\int_{t_{1}}^{t_{2}}\|\dot{\boldsymbol{q}}(t)\| \mathrm{d} t\right) /\left(\int_{t_{1}}^{t_{2}}\|\dot{\boldsymbol{\theta}}(t)\| \mathrm{d} t\right),
$$

and gives a measure for the velocity enlargement between motor and link side motion. This criterion can be maximized resulting in optimal values for the joint stiffness $\boldsymbol{K}$.

Aiming high embodiment, the criterion (24) has to be maximized and criterion (23) has to be minimized. Beside this reciprocal property, both criteria have quite similar meanings. This will be validated in Section 5 .

\subsection{Properties of the one-mode dynamics}

Shaping the nonlinear flexible joint model (13)-(15) to a one-mode dynamics results in a closed-loop system with mentionable properties:

- The closed-loop dynamics are exactly linear (cf. (21)). This implies the one-mode property (otherwise $\lambda_{i}=$ $\left.f\left(\boldsymbol{q}_{0}\right)\right)$.

- The one-mode property allows to excite the system in arbitrary directions of the joint space, always resulting in oscillations along the first mode, i.e. synchronous motion and energy transfer.

\section{SIMULATION AND EXPERIMENTAL RESULTS}

In this section, the trajectory generation approach will be validated for a planar two-link serial elastic actuated (SEA) robot manipulator via simulation and experiment.

\subsection{SEA setup}

The SEA manipulator consists of two rigid links, which are connected to each other and to the base by rotational joints (double pendulum). Each DoF of the joints is actuated by one motor, where the motor motion is transformed by a gear box and the torque acts via a linear spring on the next link. This results in a serial structure of motor, gear box, spring, and link (see Fig. 2). A prototypical hardware implementation was set up, see Fig. 3. The motor positions $\boldsymbol{\theta}$ and the link side positions $\boldsymbol{q}$ are measured by hall sensors and the time derivatives are computed by numerical differentiation. Thus, if the values of joint stiffness $\boldsymbol{K}$ and joint damping $\boldsymbol{D}$ are known, the full state of (13)-(15) can be obtained.

In the model (13)-(15), the coupling between motor and link side inertia is neglected. This means, that the rotational energy of the motor rotors is only determined by the rotor's relative motion to the previous link (Ott (2008)). Due to the high gear ratios of about 100 this assumption is valid for our setup.

\begin{tabular}{|l|l|l|}
\hline \multicolumn{2}{|c|}{ Link 1 } \\
\hline \multicolumn{1}{|c|}{} \\
\hline Gear \\
\hline Motor \\
Mounting
\end{tabular}

Fig. 2. The robot setup sketched from the side.

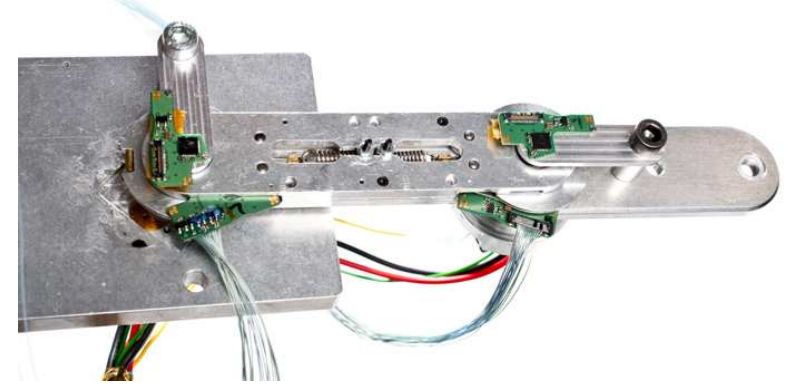

Fig. 3. Experimental setup. 
Table 1. Model parameters

\begin{tabular}{c|ccccc}
$\mathrm{i}$ & $m_{i}(\mathrm{~kg})$ & $C M_{i}(\mathrm{~m})$ & $J_{i}\left(\mathrm{kgm}^{2}\right)$ & $k_{i}(\mathrm{Nm})$ & $d_{i}(\mathrm{Nms})$ \\
\hline \hline & \multicolumn{5}{c}{ simulation model } \\
\hline 1 & 5.0 & 0.166 & 0.1386 & $100 \ldots 800$ & 8.0 \\
2 & 5.0 & 0.166 & 0.1386 & $100 \ldots 800$ & 3.2 \\
\hline & \multicolumn{5}{c}{ real hardware setup } \\
\hline 1 & 0.066 & 0.042 & 0.00021 & $2.4 \ldots 6.5$ & 0.01 \\
2 & 0.118 & 0.012 & 0.00009 & $2.4 \ldots 6.5$ & 0.01
\end{tabular}

Two parameter sets of two-link SEA manipulators are listed in Table 1. One set is used for simulation studies, and the other set is of the real hardware setup. $m_{i}, C M_{i}$ and $J_{i}$ denotes the mass, the distances to the center of mass (w.r.t. the joint axis) and the mass moments of inertia (w.r.t. the joint axis) of each link $i$. The joint stiffness $k_{i}$ can be varied by replacing the springs and the joint damping is assumed to be constant.

\subsection{Simulation results}

The range of "meaningful" embodiment was analyzed by simulations. Therefore the "simulation model" parameter set (see Table 1) was chosen, since a large variation for the mass matrix $\boldsymbol{M}\left(\boldsymbol{q}_{0}\right)$ can be generated. To excite and later again stop the oscillation, the nominal damping factor $\xi$ has been scheduled for each simulation run (see, e.g. Fig. $6(\mathrm{e}))$. The value of $\lambda_{0}=$ const. was set to the first intrinsic

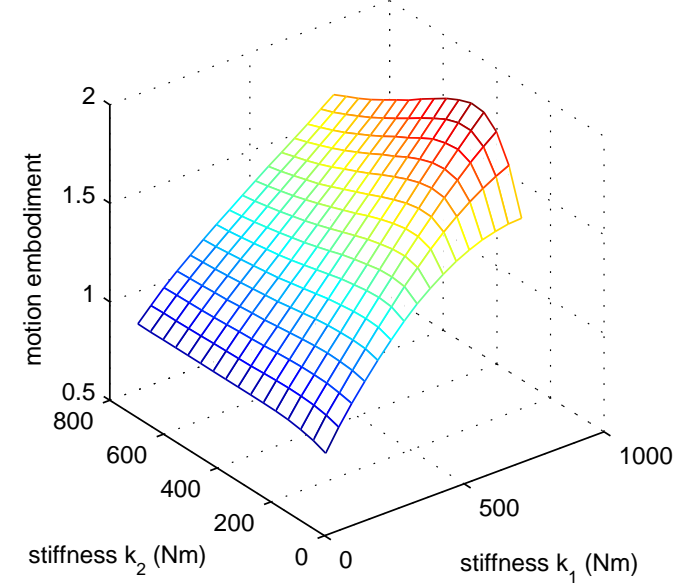

Fig. 4. Motion embodiment.

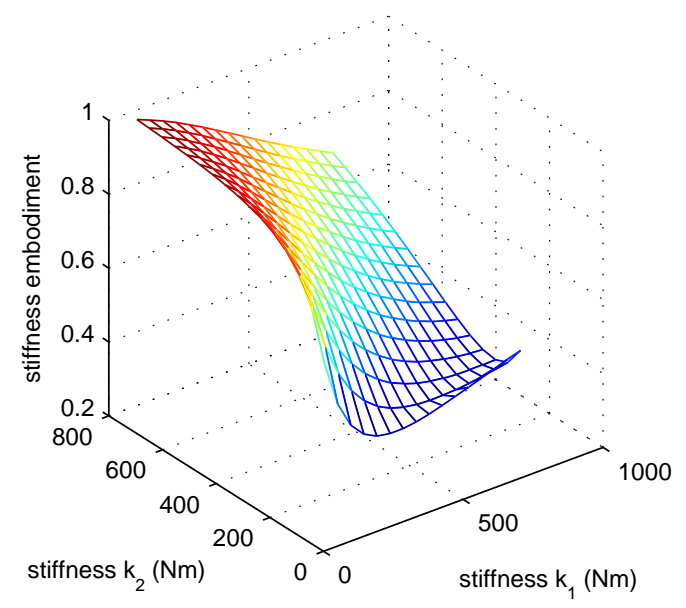

Fig. 5. Stiffness embodiment. eigenvalue for $\boldsymbol{q}_{0}=\mathbf{0}$ (see, (22)). In a first step, the criterion (24) was maximized w.r.t. a pair of joint stiffness values $\left(k_{1}, k_{2}\right)$, i.e. $\left(\bar{k}_{1}, \bar{k}_{2}\right)=\arg \max \alpha\left(k_{1}, k_{2}\right)$. This was done by sweeping all possible combinations $\left(k_{1}, k_{2}\right)$ in the range given in Table 1 . The evaluated values for the criteria (24) and (23) are plotted in Fig. 4 and 5, respectively. Then the optimal joint stiffness $\left(\bar{k}_{1}, \bar{k}_{2}\right)$ was chosen, and the damping factor in the energy inducing interval was decreased step by step to increase the oscillation amplitude in $\boldsymbol{q}$.

An example trajectory of the motor and link-side motion, together with the appropriate time series of the damping factor and the first intrinsic eigenvalue are plotted for the case of $\xi=-0.42 / \sqrt{2}$ (in the excitation interval) in Fig. 6. Under these conditions the maxima of the link-side velocities $\dot{\boldsymbol{q}}$ are slightly larger than the motor velocities $\dot{\boldsymbol{\theta}}$, and $\alpha=1.50$ and $\beta=0.47$. The variation of the first intrinsic eigenvalue is $58.3 \%$ w.r.t. the value in equilibrium position $\boldsymbol{q}_{0}=\mathbf{0}$, therefore the system is strongly nonlinear. As a result of the simulation study, the variation of $58.3 \%$ can be interpreted as a limit of the presented approach. Further increase of the variation lead to motion embodiment numbers $\alpha<1$ (i.e. the motor velocity is higher than the link-side velocity). This makes the approach unfeasible. The simulation demonstrates that the method works also in the presence of significant nonlinearities.

\subsection{Experimental results}

The method was validated on the real hardware setup by commanding the desired motor trajectory $\boldsymbol{\theta}_{d}(t)$ obtained in a offline simulation for the "real hardware setup" parameter set give in Table 1, see Fig. 7(c) and Fig. 7(d). The joint stiffness was set to the optimal combination $\left(k_{1}=7.0 \mathrm{Nm}, k_{2}=2.4 \mathrm{Nm}\right)$, obtained with the same procedure as described above. The proportional gain of the motor PD controller was set to $\boldsymbol{K}_{P}=\operatorname{diag}(1000,1000)$, and the differential gain $\boldsymbol{K}_{D}$ was chosen such that the closed loop system is critically damped. Finally, the desired controller torques $\boldsymbol{\tau}_{c}$ were directly commanded to the current controllers of the motors.

Figure 7 shows the results of the simulations and experiments for the system with the parameter set "real hardware setup" from Table $1^{3}$. The trajectory consists of three parts. First, the system is excited from rest position by a negative damping coefficient. Once the desired oscillation amplitude is reached, the damping is set to zero and the system sustains the motion. Finally, the oscillation is stopped by a positive damping factor.

As the system is designed to contain one repeated eigenvalue, the motions of the two links are exactly coincident in the simulation. The experimental measurements show very similar performance as the simulation and the position and velocity plots of the two link motions are accurately matching. The desired motor motions computed by the algorithm are different in amplitude and slightly phase shifted from the measured ones. This is as to generate the desired one-mode behaviour and excite the system,

\footnotetext{
3 Measured velocities are obtained by low pass filtering at $30 \mathrm{~Hz}$ and deriving the position measurements numerically.
} 
an adapted trajectory is necessary. In the experiment, the motor PD controller precisely tracks the desired motion.

The oscillation amplitudes which can be reached by the real hardware setup are restricted trough maximal elongation of the springs. Thus, the behavior shown in Fig. 7 is quasi-linear. As shown in the simulation study (see. Fig. $6)$, this is only a limitation of the current hardware setup and not a restriction of the method.

A video of the experiments can be found at https: //www . robotic.dlr.de/index.php?id=337.

\section{DISCUSSION AND CONCLUSION}

\subsection{Discussion}

The presented approach has several advantages. The onemode shaping and negative damping excitation allows to beneficially exploit the elastic elements as not only motion is embodied but even the system is operated where it has the least damping. During several oscillations, the motor can provide power to the system, which is stored as elastic and kinetic energy. Therefore the resonant dynamics allow to extend the energy transformation process for time periods longer than the intrinsic eigenfrequency of the system. As a result the maximum system energy is only limited by hardware maximum ratings (e.g. system end stops, load capability) and not by power restrictions of the energy source.

However, if the natural system properties are very different from the desired properties, the system inputs (motors) have to shape the dynamics. Therefore, the system performance is limited by the embodiment of the desired behaviour into the robot.

As a result of the method, the synchronized energy transfer between the joints and the only-first-modes dynamics allow to identify points in the resulting trajectory at which all the energy of the system is potential or kinetic. For example the passive springs are relaxed at zero crossings of the link position and all the energy is kinetic.

Furthermore, the reduction from $n$-eigenmodes to only one eigenmode provides an abstraction mechanism which allows to extend methods which are designed for singleDoF systems and use them on multi-DoF systems such as input shaping (an approach using feedback linearization was presented in Banerjee and Singhose (1998)).

This approach aims to benefit from the intrinsic properties of robots with elastic joints. Therefore, the work to be done is substantiated by the three major steps:

(1) Embodiment and excitation of oscillations in serial elastic actuated robot manipulators (with more than one DoF);

(2) Handling of the variations due to the nonlinear mass matrix and compensation for the Coriolis/centrifugal forces;

(3) Extending the method for variable stiffness actuated (VSA) robots with nonlinear spring characteristics.

Since the first two points are already solved in this paper, further investigations will concentrate on the extension for the general case of VSA robots.

\subsection{Conclusion}

This work presents an approach to exploit the intrinsic dynamics of a robot with mechanical elastic elements. The idea is to shape the system such that a coordinated behaviour arises while the motion is embodied and the natural dynamic is used. Therefore, a desired system is formulated which contains one repeated eigenmode and makes use of the intrinsic eigenmodes of the linearized robot dynamics. To excite the oscillatory system, negative damping is implemented as energy input. A controller is developed which allows to apply the approach to systems that can be modeled by the reduced flexible joint model. Since the complete system can be separated in two uncoupled subsystems, asymptotic stability is proven for both subsystems: the trajectory generator and the controlled robot-resulting in stability for the whole system. Furthermore, simulations and experiments on a SEA double pendulum are conducted showing the effectiveness of the approach.

\section{REFERENCES}

Albu-Schäffer, A. and Hirzinger, G. (2000). State feedback controller for flexible joint robots: A globally stable approach implemented on dlr's light-weight robots.

Albu-Schäffer, A., Wolf, S., Eiberger, O., Haddadin, S., Petit, F., and Chalon, M. (2010). Dynamic modelling and control of variable stiffness actuators. In Proc. IEEE Int. Conf. on Robotics and Automation.

Banerjee, A.K. and Singhose, W.E. (1998). Command shaping in tracking control of a two-link flexible robot. Journal of Guidance, control, and Dynamics, 21, 10121015.

Braun, D.J., Howard, M., and Vijayakumar, S. (2011). Exploiting variable stiffness in explosive movement tasks. In Robotics: Science and Systems.

Brooks, R.A., Breazeal, C., Marjanovic, M., Scassellati, B., and Williamson, M.M. (1999). The cog project: Building a humanoid robot. Lecture Notes in Computer Science, $1562,52-87$.

De Luca, A., Siciliano, B., and Zollo, L. (2005). Pd control with on-line gravity compensation for robots with elastic joints: Theory and experiments. Automatica, 41(10), 1809 - 1819.

Grebenstein, M., Albu-Schäffer, A., Bahls, T., Chalon, M., Eiberger, O., Friedl, W., Gruber, R., Haddadin, S., Hagn, U., Haslinger, R., Höppner, H., Jörg, S., Nickl, M., Nothhelfer, A., Petit, F., Reill, J., Seitz, N., Wimböck, T., Wolf, S., Wüsthoff, T., and Hirzinger, G. (2011). The DLR Hand Arm System. In Proc. IEEE Int. Conf. on Robotics and Automation.

Haddadin, S., Huber, F., and Albu-Schäffer, A. (2012). Optimal control for exploiting the natural dynamics of variable stiffness robots. IEEE International Conference on Robotics and Automation (ICRA2012), Minneapolis, USA.

Jafari, A., Tsagarakis, N.G., and Caldwell, D.G. (2011). A novel intrinsically energy efficient actuator with adjustable stiffness. ASME Transactions on Mechatronics.

Khalil, H. (2002). Nonlinear Systems, 3rd edn. Prentice Hall, Englewood Cliffs. 


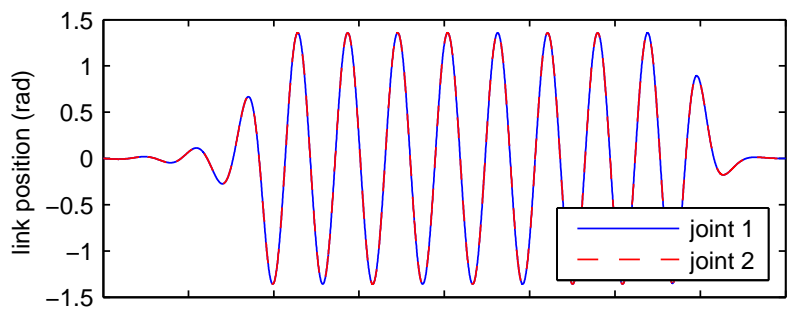

(a)

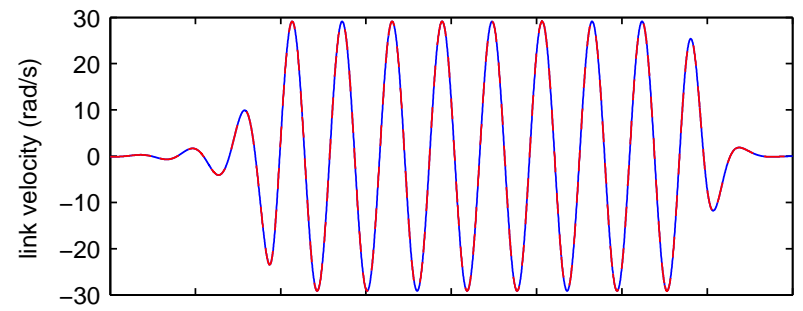

(c)

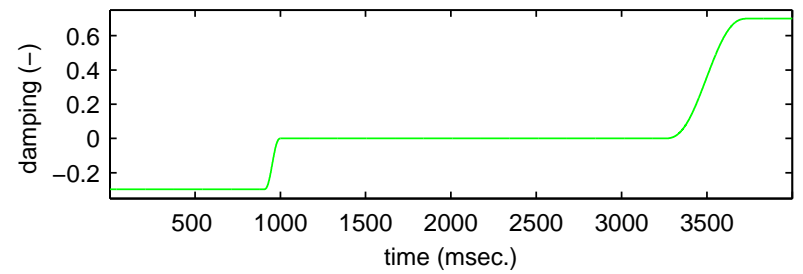

(e)

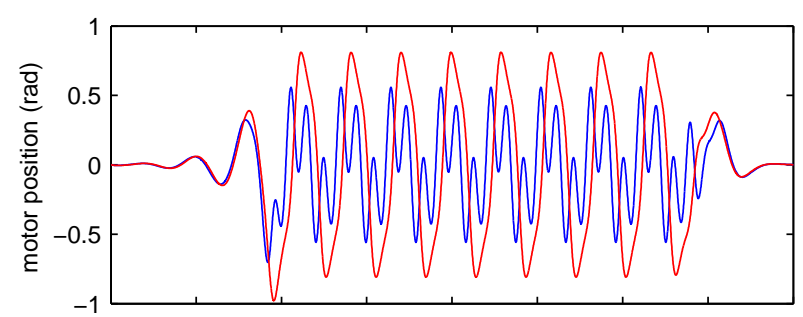

(b)

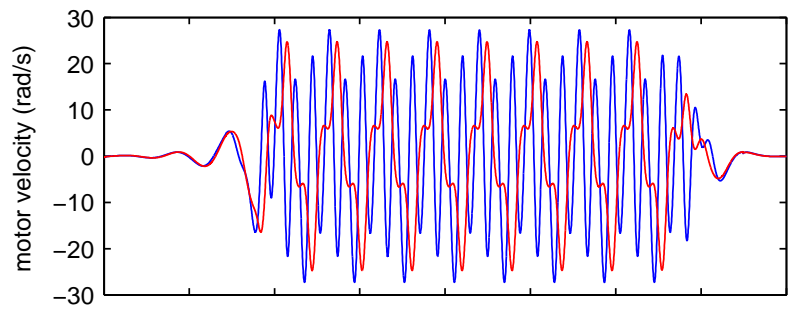

(d)

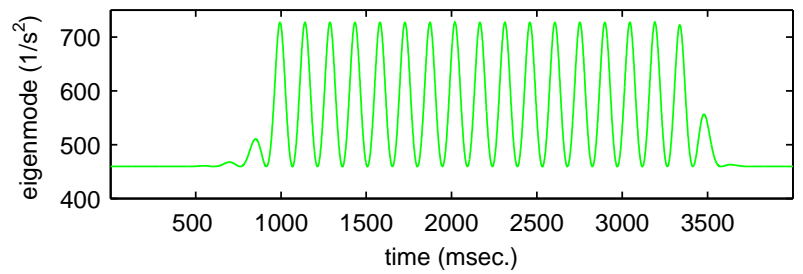

(f)

Fig. 6. Simulation results validating the feasible range for variation of the first intrinsic eigenvalue. For these conditions the motion embodiment measure $\alpha=1.50$, and the stiffness embodiment measure $\beta=0.47$. The variation of the first intrinsic eigenvalue is $58.3 \%$ w.r.t. the value in equilibrium position. The amplitudes of the link velocities are slightly larger than these of the motor velocities.

Kokotovic, P., Khalil, H., and OReilly, J. (1986). Singular Perturbation Methods in Control: Analysis and Design. Academic Press, London.

Nicosia, S. and Tomei, P. (1992). A method to design adaptive controllers for flexible joint robots. In Proc. IEEE Int. Conf. on Robotics and Automation.

Oh, J.H. and Lee, J.S. (1997). Control of flexible joint robot system by backstepping design approach. In Proc. IEEE Int. Conf. on Robotics and Automation.

Oh, J.H. and Lee, J.S. (1998). Backstepping control design of flexible joint manipulator using only position measurements. In Proc. IEEE Int. Conf. on Robotics and Automation.

Ott, C. (2008). Cartesian Impedance Control of Redundant and Flexible-Joint Robots. Springer.

Pratt, G.A. and Williamson, M.M. (1995). Series elastic actuators. In Proc. of the IEEE/RSJ International Conference on Intelligent Robots and Systems., 399-406. Pittsburg, PA, USA.

Raibert, M. (1986). Legged Robots that Balance. MIT Press.
Spong, M.W. (1987). Modeling and control of elastic joint robots. Transactions of the ASME: Journal of Dynamic Systems, Measurement, and Control, 109, 310-319.

Tonietti, G., Schiavi, R., and Bicchi, A. (2005). Design and control of a variable stiffness actuator for safe and fast physical human/robot interaction. In Proc. IEEE Int. Conf. on Robotics and Automation, 528-533.

Uemura, M. and Kawamura, S. (2009). Resonance-based motion control method for mulit-joint robot through combining stiffness adaptation and iterative learning control. In Proc. IEEE Int. Conf. on Robotics and Automation.

Visser, L.C., Stramigioli, S., and Bicchi, A. (2011). Embodying desired behavior in variable stiffness actuators. In 2011 Congress of the International Federation of Automatic Control - IFAC 2011.

Wolf, S. and Hirzinger, G. (2008). A new variable stiffness design: Matching requirements of the next robot generation. In Proc. IEEE Int. Conf. on Robotics and Automation. 


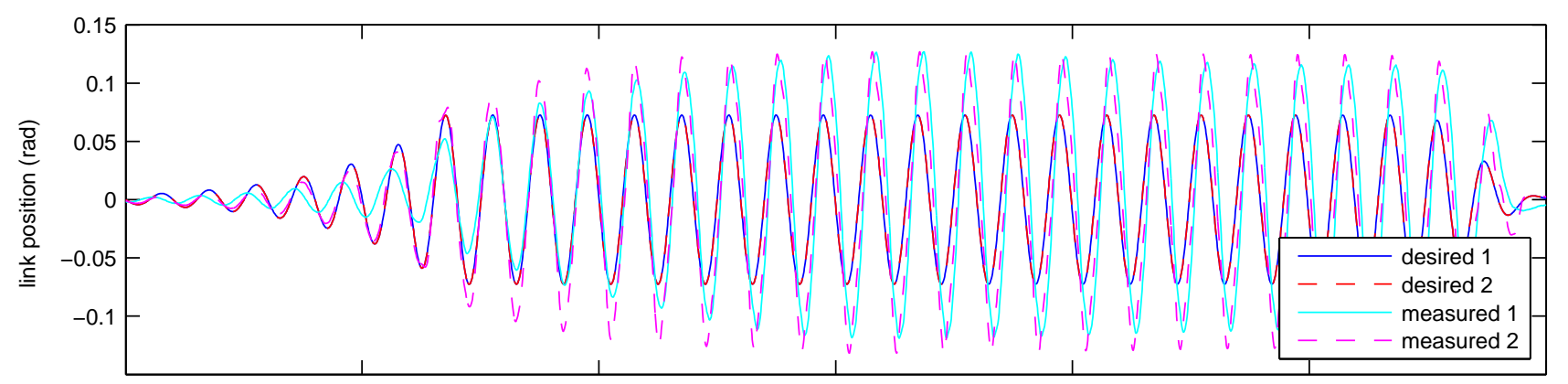

(a)

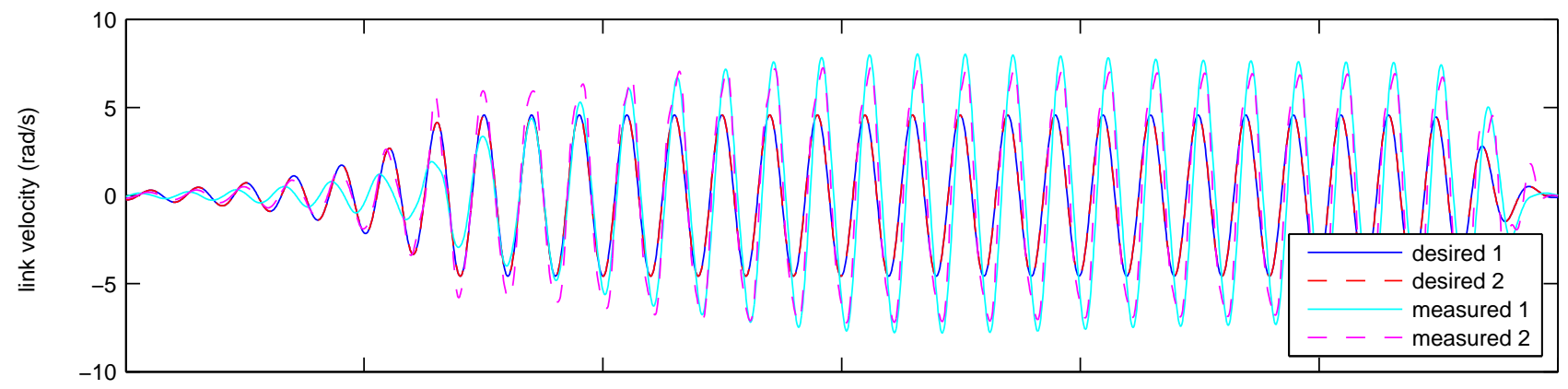

(b)

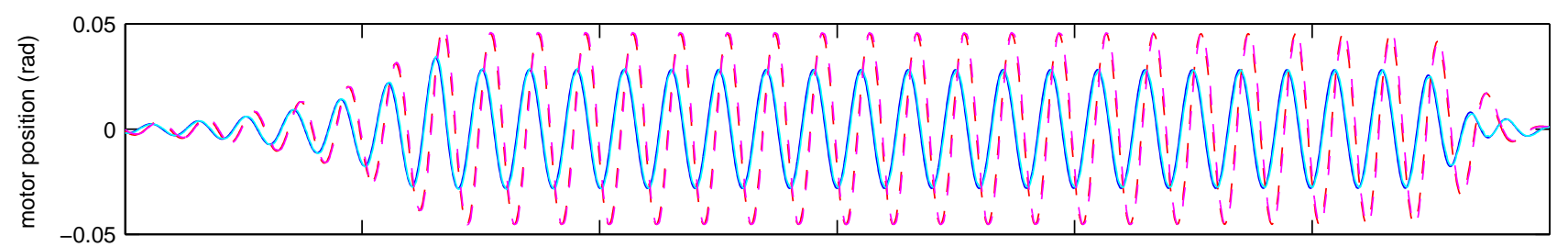

(c)

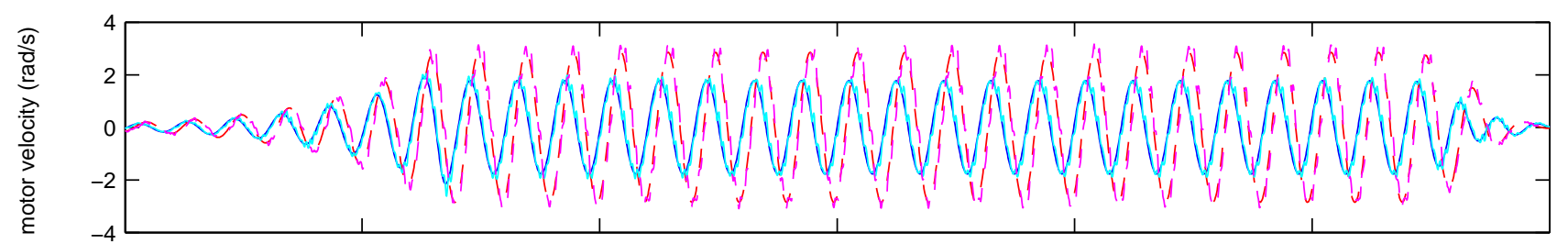

(d)

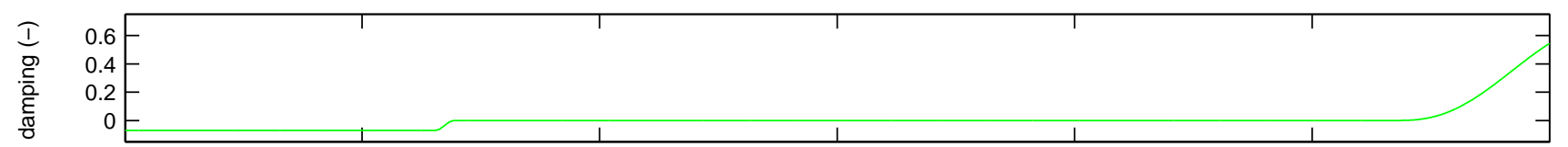

(e)

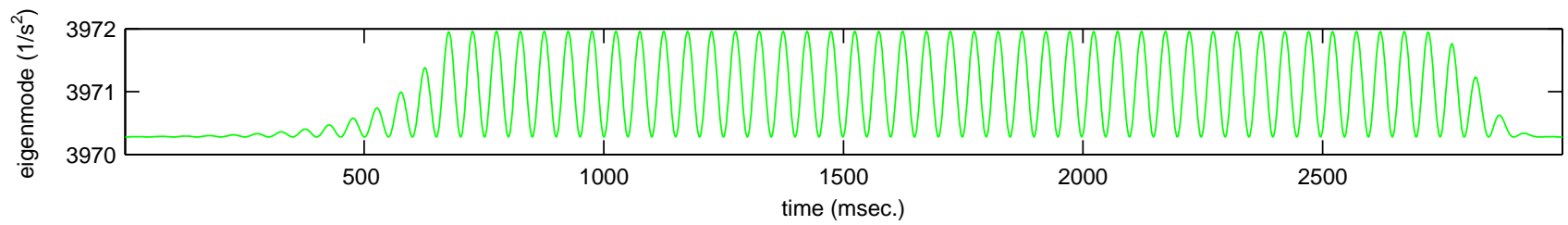

(f)

Fig. 7. Comparison of the simulated and measured trajectories. The measurements show very good matching of the predicted and achieved behaviour. The motion of the two links coincides precisely. The two motor trajectories are slightly different as they contain elements which adapt the system behaviour to the desired one mode property and excite the oscillations. The three motion parts oscillation excitation, oscillation conservation and damping are induced by the damping factor. 